\title{
Evolución de la calidad de frutos de butiá (Butia capitata, Mart. Becc.) en almacenamiento refrigerado
}

\section{Quality evolution of butiá fruits (Butia capitata, Mart. Becc.) in refrigerated storage}

\author{
$\underline{\text { Zaccari, Fernanda }}^{(1)}$, Crosa, María José ${ }^{(2)}$, Irisity, Mariana ${ }^{(3)}$ \\ (1) Grupo Poscosecha de Frutas y Hortalizas, Facultad de Agronomía, Universidad de la República, Uruguay - (2) Gerencia \\ de Proyectos Alimentarios, Laboratorio Tecnológico del Uruguay, LATU, Uruguay - ${ }^{(3)}$ Departamento de Gestión y Trans- \\ ferencia Tecnológica para el Desarrollo Local. Laboratorio Tecnológico del Uruguay, LATU, Uruguay.
}

Contacto: fzaccari@fagro.edu.uy

Recibido: 12/6/2012 - Aprobado: 26/9/2012

\begin{abstract}
$\underline{\text { Resumen }}$
El objetivo de este trabajo fue evaluar la evolución de la calidad de frutos de butiá, cuantificar e identificar las principales causas de su deterioro, y caracterizarlos física y químicamente conservados a 5,15 y $20{ }^{\circ} \mathrm{C}$ durante $0,7,21$ y 28 días. A la semana de almacenamiento el $47 \%, 24 \%$ y $22 \%$ del peso de frutos no presentaba defectos visibles a 5,15 y 20 ' $\mathrm{C}$, respectivamente. A los 21 días a 15 y $20^{\circ} \mathrm{C}$ se observó pudriciones y manchas en todos los frutos. A mayor temperatura y tiempo de conservación aumentó el tono del color de la pulpa $\left(76,7\right.$ a $80,5^{\circ}$ hue). En cáscara no se encontró efecto de temperatura ni del tiempo de almacenamiento en el contenido de materia seca (MS, $21,1 \%$ y trans- $\beta$-caroteno $(1,96 \mathrm{mg} / 100 \mathrm{~g}$ fresco). En la pulpa el contenido de trans- $\beta$-carotenos/100 g aumentó de 1,06 a 1,15 mg. Los sólidos solubles totales (SST) y la acidez titulable (AT) del jugo disminuyeron con el tiempo de conservación: 14,8 a $10,1{ }^{\circ} \mathrm{Brix}$ y 1,60 a $1,37 \%$ ácido cítrico, respectivamente. En las condiciones de este estudio se concluye que el butiá almacenado a $5^{\circ} \mathrm{C}$ durante 7 días mantiene un $47 \%$ de los frutos sin defectos y con aptitud para su consumo in natura o industrializado.

Palabras clave: Butia capitata, fruto nativo, poscosecha.
\end{abstract}

\begin{abstract}
The aim of this study was to quantify and identify the main causes of butia fruits deterioration and to characterize physical and chemically composition changes during their storage at 5,15 and $20{ }^{\circ} \mathrm{C}$ for $0,7,21$ and 28 days. After a storage week $47 \%, 24 \%$ and $22 \%$ of fruits (by weight) did not show visible defects at 5,15 and $20{ }^{\circ} \mathrm{C}$, respectively. After 21 days, all the fruits were rotted and stained when stored at temperatures of 15 and $20^{\circ} \mathrm{C}$. The higher temperature and storage time increased the pulp color tone of the fruits $\left(76,7\right.$ to $80,5^{\circ}$ hue). The effects of skin temperature or time storage were null both on dry matter (DM, 21,1\%) or trans- $\beta$-carotene $(1,96 \mathrm{mg} / 100 \mathrm{~g}$ fresh) fruit contents. The trans- $\beta$-carotene/100 $\mathrm{g}$ content in pulp increased from 1,06 to 1,15 mg. Soluble solids (STT) and titratable acidity of juice decreased during storage time from 14,8 to $10,1{ }^{\circ}$ Brix and 1,60 to 1,37 (\% citric acid), respectively. Under study conditions it is concluded that $47 \%$ of butia fruits remained without defects for consumption in natura or processed when stored for 7 days at $5^{\circ} \mathrm{C}$.

Keywords: Butia capitata, native fruit, postharvest.
\end{abstract}

\section{Introducción}

Las palmas de Butia capitata (Mart.) Becc. son parte de la Reserva de Biosfera Bañados del Este Programa MAB, 1976 (Rivas y Barilani, 2004). Estas palmas forman un ecosistema particular con alto valor paisajístico, cultural y socio económico, y sus riesgos y amenaza de extinción han sido ampliamente reportados (Báez y Jaurena, 2000; Rivas y Barilani, 2004). Las palmas tienen más de 300 años y están ubicadas dentro de sistemas de producción ganadero y agrícola (arroz y soja), en los cuales se hace muy difícil su reposición natural (Báez y Jaurena, 2000; Rivas, 2001). Se han realizado varios estudios, trabajos de investigación y de capacitación para frenar la pérdida del palmar y a la vez promover su regeneración y la elaboración de nuevos productos (Rivas 2001; Crosa, 2011).
Los frutos de butiá son consumidos in natura e industrializados artesanalmente por los habitantes de la región (Betancurt et al., 2008; Crosa et al., 2011; Burzaco et al., 2011). En las condiciones tradicionales de cosecha, almacenamiento y procesamiento se ha constatado una pérdida muy rápida de la calidad de los frutos que aún no ha sido cuantificada. Una propuesta conducente a preservar las palmas y la biodiversidad de su ecosistema es encontrar sistemas de explotación racional en los cuales esté incluido un procedimiento de colecta y de almacenamiento de los frutos in natura o con algún grado de elaboración.

Estos frutos silvestres tienen propiedades de interés nutricional, funcional y medicinal atribuida a compuestos antioxidantes, como polifenoles, Vitamina C, Vitamina A y fibra alimentaria (Schwartz et al., 2007; Faria et al., 2008, Crosa et al., 2011). La técnica utilizada en la cosecha y en poscosecha de los frutos son factores determinantes de 
la calidad composicional y nutricional de los butiá para su consumo directo, así como de la materia prima que se utiliza para la obtención de subproductos.

Este trabajo tuvo como objetivos: caracterizar la conservación de frutos de butiá in natura almacenados en tres temperaturas, $5,15 \mathrm{y}$ $20{ }^{\circ} \mathrm{C}$, durante $0,7,21$ y 28 días, identificar las principales causas de deterioro y cuantificar la evolución del color, el contenido de materia seca, trans- $\beta$-caroteno, sólidos solubles totales, $\mathrm{pH}$ y acidez titulable expresado en ácido cítrico en la pulpa y cáscara.

\section{Materiales y Métodos}

\section{Material vegetal}

Los frutos fueron obtenidos en la "Vuelta del Palmar" en el área de Castillos, Rocha, Uruguay. Se seleccionaron las palmas que presentaban frutos de butiá de color anaranjado según Pantone 152C (L 49,6; ${ }^{\circ}$ Hue 64,6; Croma 58,9) y de ellas se cosecharon los frutos que no se desprendieron del racimo durante el proceso del corte y caída. Los frutos cosechados en abril de 2011 fueron trasladados a LATU (Montevideo) donde se lavaron y desinfectaron. La desinfección consistió en inmersión de los frutos en agua con hipoclorito de sodio $(\mathrm{NaClO}, 100 \mathrm{ppm})$ durante $5 \mathrm{~min}$. Luego se enjuagaron y se seleccionaron frutos sin daños, pudriciones y/o alteraciones visibles (Figura 1).

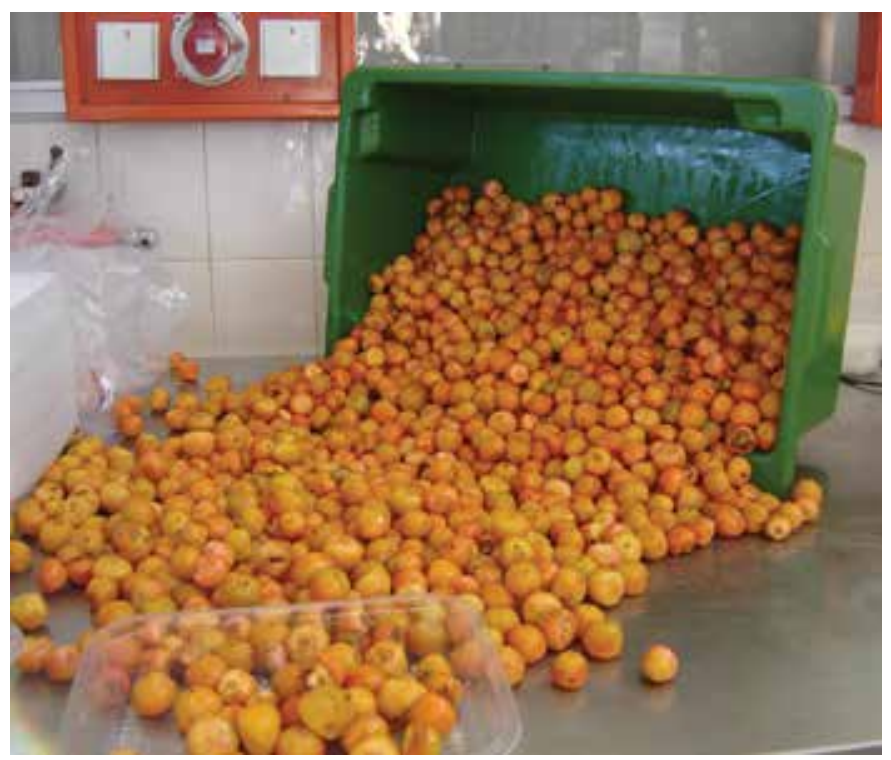

Figura 1. Selección de frutos para almacenar.

Los frutos sin defectos fueron colocados en bandejas de PVC al azar ( 50 frutos por repetición por tratamiento). Las bandejas fueron almacenadas durante 7, 21 y 28 días dentro de cámaras acondicionadas a tres temperaturas 5,15 y $20^{\circ} \mathrm{C}$ y a $90 \% \mathrm{HR}$. Se utilizaron tres repeticiones (bandejas) para cada tratamiento para las evaluaciones realizadas.

\section{Pérdida de peso}

Al instalar el estudio ( 0 días) y en cada momento de salida de cámara $(7,21$ y 28 días) se determinó el peso total de los frutos y el peso de frutos sin defectos y con defectos visibles en una balanza digital (Shimadzu, Modelo: Bx $22 \mathrm{KH}, 20000 \mathrm{~g} \pm 0.1 \mathrm{~g}$ ). Se calculó la pérdida de peso durante el almacenamiento con la siguiente ecuación:
$\%$ pérdida de peso $=$ [peso a la salida de cámara $] \times$ [peso inicialmente almacenado] ${ }^{-1}$ x 100

\section{Determinación de defectos en la calidad en la fruta}

En cada salida de cámara se cuantificó el número y el peso de frutos sin defectos y con defectos. Los defectos identificados fueron frutos con pudriciones, manchados y arrugados y se expresaron como porcentaje del peso inicialmente almacenado. A fin de identificar los patógenos causantes de las pudriciones se enviaron muestras al Laboratorio de Diagnóstico de Enfermedades de la Facultad de Agronomía (Universidad de la República, Montevideo).

\section{Color en cáscara y pulpa}

El color fue medido en 20 frutos en la zona ecuatorial en zonas opuestas, por duplicado, para la cáscara y la pulpa en cada salida de cámara y para cada temperatura de conservación. La medición se realizó por el sistema CIE $\mathrm{L}^{*} \mathrm{a} * \mathrm{~b}^{*}$, utilizando un colorímetro trí-estimulo (Minolta CR-10, Japón). Se midieron las variables L* (Luminosidad, $0=$ negro a $100=$ blanco $), a^{*}(-a=$ verde, $a=$ rojo $)$ y b* (-b $=$ amarillo, $b=a z u l)$. Se calculó la saturación del color (Croma), el tono (ángulo Hue, ${ }^{\circ}$ hue) y el índice de color (IC) con las siguientes ecuaciones (Jiménez et al., 1981):

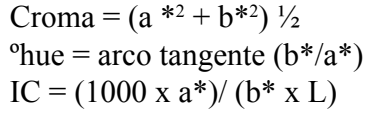

\section{Contenido de materia seca}

Se pesó una muestra fresca de $5 \mathrm{~g}$ de pulpa o cáscara y se secó en estufa (Blue.M., EUA) con ventilación cenital a $105{ }^{\circ} \mathrm{C}$ hasta peso constante. La medición se realizó por triplicado. Se calculó el porcentaje de materia seca $(\% \mathrm{MS})$ de cada muestra de la siguiente manera:

Materia Seca $(\%)=($ peso seco muestra $) \times(\text { peso fresco muestra })^{-1}$ $\mathrm{x} 100$

\section{Contenido de sólidos solubles totales}

Los sólidos solubles totales (SST) se determinaron en la pulpa de 20 frutos con un refractómetro digital (ATAGO Poquet PAL-10 a 53 $\pm 0,1^{\circ}$ Brix, Japón). Las mediciones se realizaron en el jugo extraído de la pulpa por compresión. Los valores se expresaron en ${ }^{\circ}$ Brix sin corrección por acidez.

\section{Determinación del pH y la acidez titulable del jugo}

Se obtuvo el jugo de la pulpa de 20 frutos sobre el cual se determinó por triplicado el $\mathrm{pH}$ y la acidez titulable (AT). El pH del jugo fue medido mediante un pHmetro digital $\left( \pm 0,01\right.$; DigySense ${ }^{\circledR}$ Cole-Parmer). La acidez titulable fue determinada en una muestra de $10 \mathrm{~mL}$ de jugo, por triplicado, con una solución de $\mathrm{NaOH}$ 0,1 N. Los resultados fueron expresados en \% de ácido cítrico.

\section{Determinación del contenido de trans- $\beta$-caroteno}

La cuantificación de trans- $\beta$-caroteno fue realizada de acuerdo a Szpylka y De Veris (2005) con modificaciones (Zaccari, 2010). Para la extracción se utilizó $1 \mathrm{~g}$ de pulpa y $0,5 \mathrm{~g}$ de cáscara en una solución de extracción de tetrahidrofurano y metanol $(25: 75 \mathrm{v} / \mathrm{v})$. 
El procedimiento se repitió hasta pérdida total de color en la muestra. La cantidad de trans- $\beta$-caroteno se cuantificó por cromatografía líquida de alta resolución (HPLC, Spectral Series P100, Thermo Separations Products, EUA), utilizando el estándar externo trans- $\beta$-caroteno tipo I (Sigma-Aldrich), una columna C30 YMCTMCarotenoid S-5; 4,6 $\mu \mathrm{m} \times 250 \mathrm{~mm}$ (Waters, EUA) termostatizada a $30^{\circ} \mathrm{C}$, y fase móvil de etanol, metanol y tetrahidrofurano $(75: 20: 5 \mathrm{v} / \mathrm{v} / \mathrm{v})$. La detección se realizó en longitud de onda de $450 \mathrm{~nm}$ con un detector UV-visible (UV-2000 SpectralSytem ${ }^{\circledR}$, EUA). Los resultados se expresaron en $\mathrm{mg}$ de trans- $\beta$-caroteno $/ 100 \mathrm{~g}$ peso fresco.

\section{Análisis Estadístico}

El diseño experimental consistió en un diseño factorial de tres temperaturas (Temperatura) y cuatro tiempos de almacenamiento (Momento). Los datos obtenidos en la variable de pérdida de peso se estudiaron mediante un análisis de varianza (ANAVA; $\leq 0,05$ ), y en el caso que correspondió, las medias se separaron por test de Tukey ( $\mathrm{P}$ $\leq 0,05$ ). Dentro de cada temperatura y momento de almacenamiento, para las variables color, MS, STT, AT, $\mathrm{pH}$ y trans- $\beta$-caroteno se realizó un ANAVA de una vía, y en el caso que correspondió se separaron las medias por el test de Tukey $(\mathrm{P} \geq 0,05)$. Los análisis estadísticos fueron procesados en el programa InfoStat (2004)/Versión 2007p.

\section{Resultados y Discusión}

\section{Pérdida de peso}

En la Figura 2 se observa que los frutos "sanos", sin defectos visibles, conservados a $5{ }^{\circ} \mathrm{C}$ presentaron una pérdida diaria de peso cercano al $1 \%$ del peso inicial y un $4 \%$ en frutos almacenados a 15 y $20^{\circ} \mathrm{C}$.

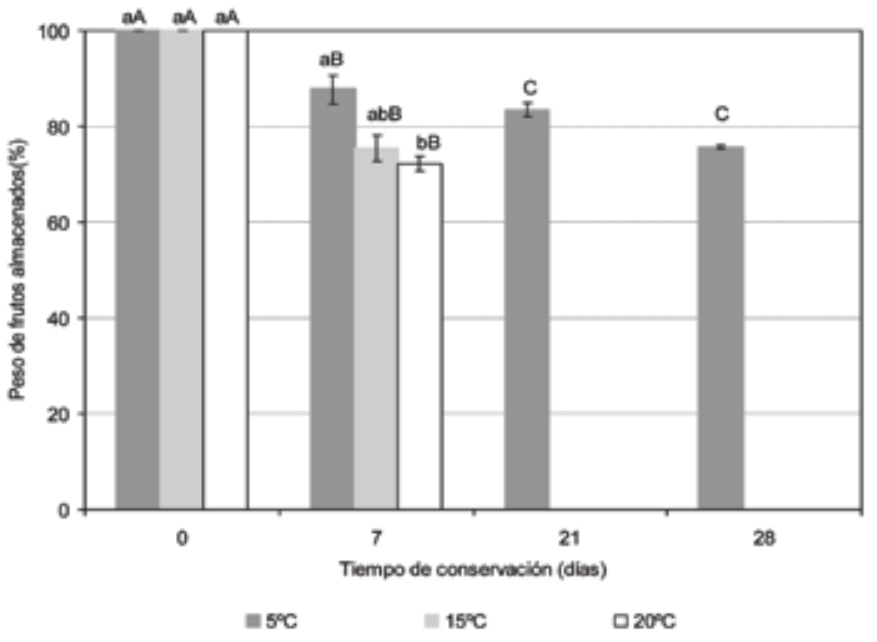

Figura 2. Pérdida de peso de los frutos sin defectos durante la conservación a $5^{\circ} \mathrm{C}, 15^{\circ} \mathrm{C}$ y $20^{\circ} \mathrm{C}$ durante 7,21 y 28 días. Media $\pm E E$. Letras minúscula iguales dentro de cada momento de evaluación indican que no hay diferencias estadísticas (Tukey $\geq 0,05$ ). Letras mayúsculas iguales para cada temperatura de conservación indican que no hay diferencias estadísticas entre momentos de evaluación (Tukey $\geq 0,05$ ).

Todos los frutos fueron descartados por pudrición, manchas $\mathrm{u}$ arrugamientos a los 21 y 28 días conservados a 15 y $20^{\circ} \mathrm{C}$ (Figura 3 y 4 A). Estos resultados verificaron una alta tasa de deterioro de los frutos de butiá al incrementarse la temperatura a $20{ }^{\circ} \mathrm{C}$ (Salunkhe y Desai, 1984), coincidente con los resultados de Amarante y Megguer (2008). Estos autores determinaron una tasa de respiración en frutos de Butia eriosphata (Martius) almacenados a $0{ }^{\circ} \mathrm{C}$ de $50,26 \mathrm{nmol} \mathrm{CO}$, $\mathrm{kg}^{-1} \mathrm{~s}^{-1}$ y 10,7 veces superior a $20^{\circ} \mathrm{C}$, con un cociente de temperatura $\left(\mathrm{Q}_{10}\right)=4,3$ cuando la temperatura se incrementó de $0{ }^{\circ} \mathrm{C}$ a $10^{\circ} \mathrm{C}$, y un $\mathrm{Q}_{10}=2,5$ al pasar de 10 a $20^{\circ} \mathrm{C}$.

\section{Defectos de calidad en la fruta}

Las pudriciones por hongos, frutos manchados y arrugados fueron las tres causas de deterioro de la calidad de la fruta y de los descartes en este estudio (Figuras 3 y 4). En frutos conservados a 15 y $20^{\circ} \mathrm{C}$ se observó la presencia de larvas de gorgojos y mosca. Los huevos de estos insectos estaban en el interior del coquito y/o pulpa, no fueron detectados síntomas en el momento de instalar el estudio, y se desarrollaron durante el almacenamiento. No se registraron pudriciones de frutos en el almacenamiento a $5{ }^{\circ} \mathrm{C}$. En esta temperatura de conservación se observó arrugamiento y ablandamiento de los frutos que no puede atribuirse a la deshidratación, dado que se mantuvo la humedad relativa ambiente alta (90-95\%) en las cámaras de conservación. Este fenómeno probablemente fue consecuencia de los procesos de deterioro ocasionado en parte por los daños en la cosecha y la alta tasa metabólica que aceleraron la senescencia de los tejidos, provocando a nivel de pared celular su colapso (Negi y Handa, 2008). Se aislaron dos hongos patógenos causantes de las pudriciones de frutos de butiá de este estudio, Penicillium sp. y otro hongo aún no reportado en el país que está siendo analizado para su identificación. Penicillium sp. es un hongo comúnmente observado como agente causal de patologías en poscosecha de frutos (Bartz y Brecht, 2003). Las manchas de color marrón que aparecieron en todas las temperaturas y tiempos de conservación puede deberse a procesos de deterioro, como oxidación enzimática y/o el inicio del desarrollo de los hongos que producen la pudrición en almacenamiento (Bartz y Brecht, 2003).

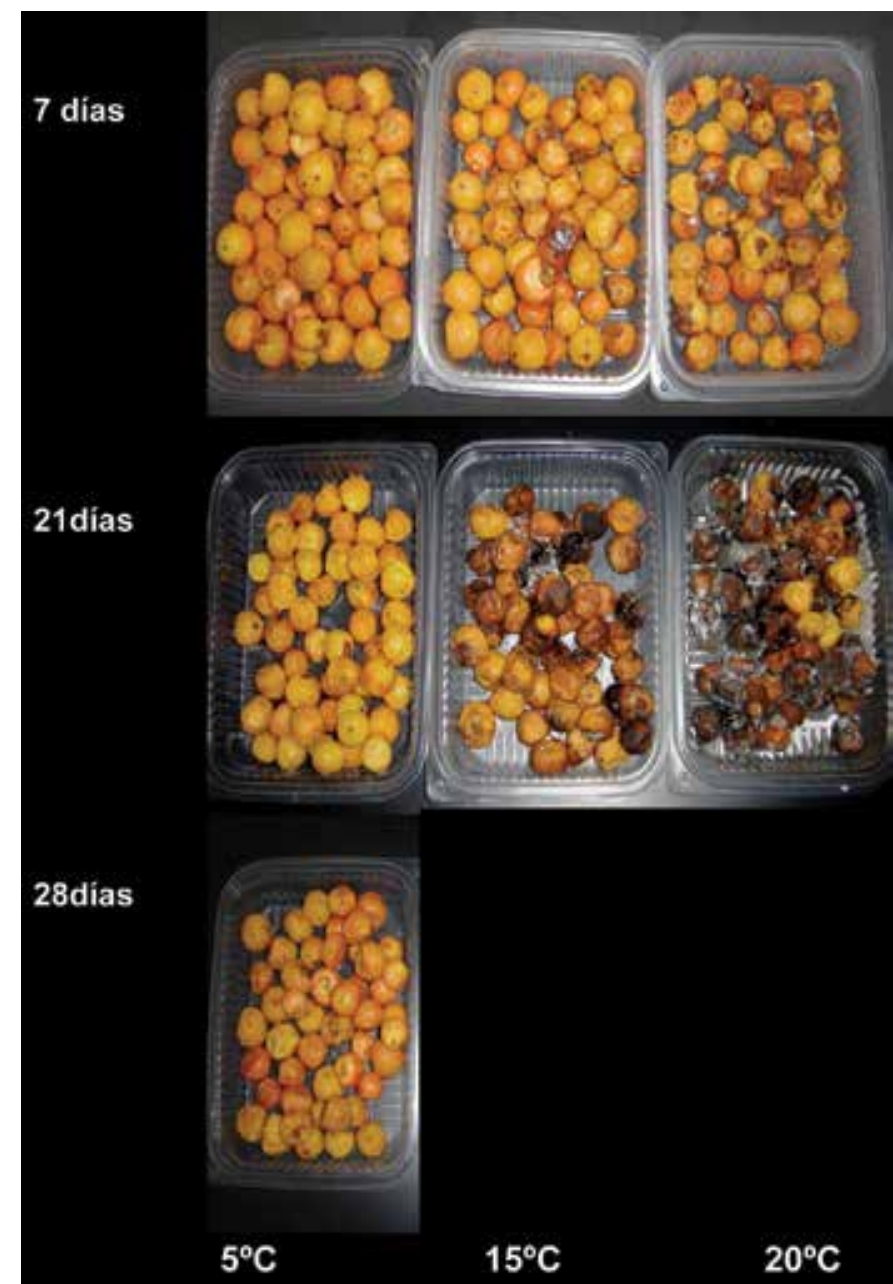

Figura 3. Frutos de butiá en el momento de salida de cámaras de almacenamiento a los 7,21 y 28 días, conservados en tres temperaturas $5 ; 15$ y $20^{\circ} \mathrm{C}$. 
A.

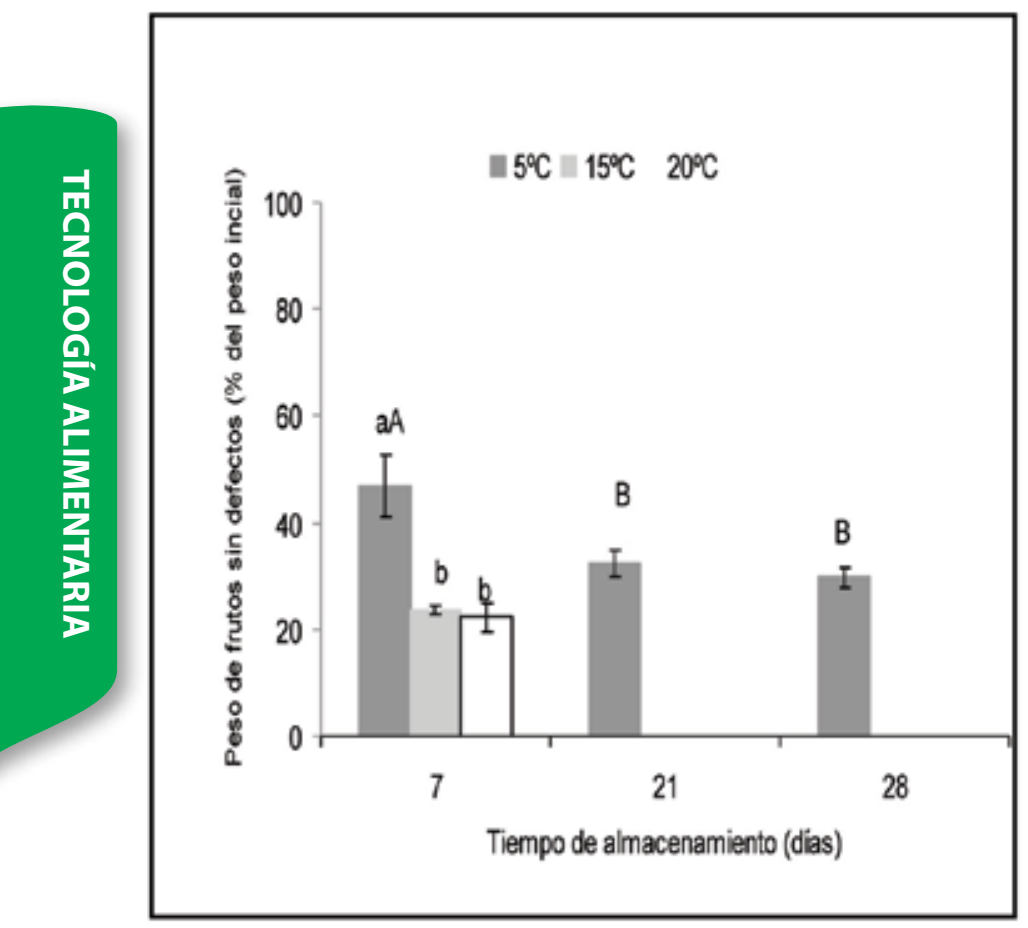

C.

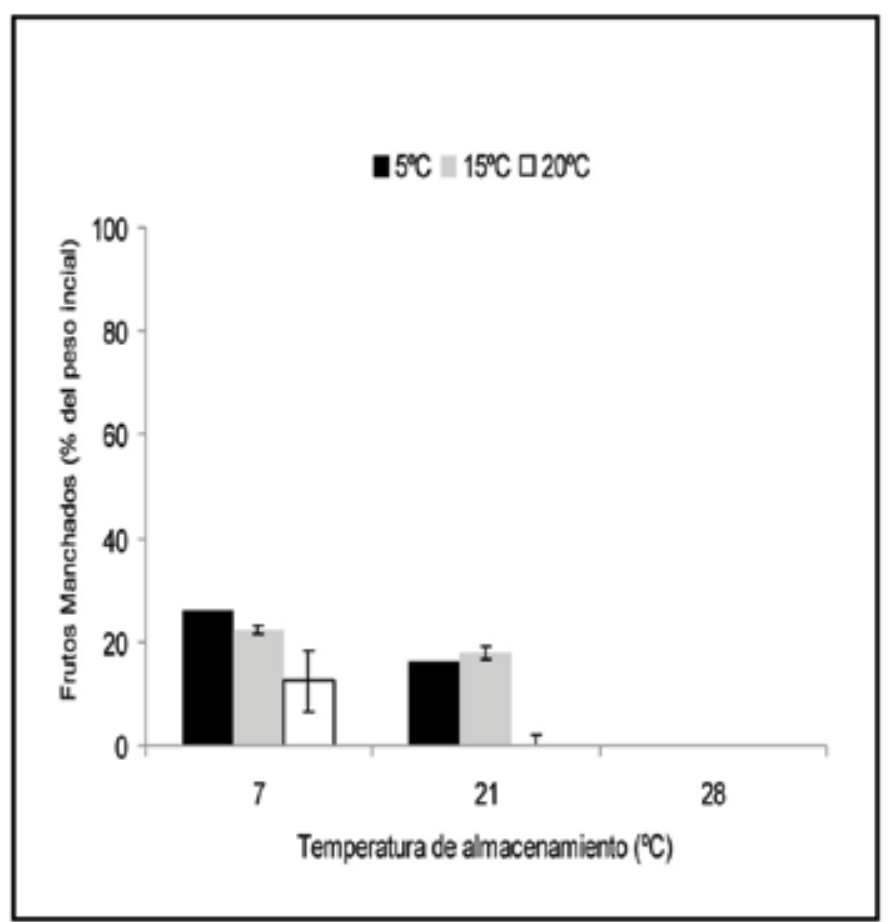

B.

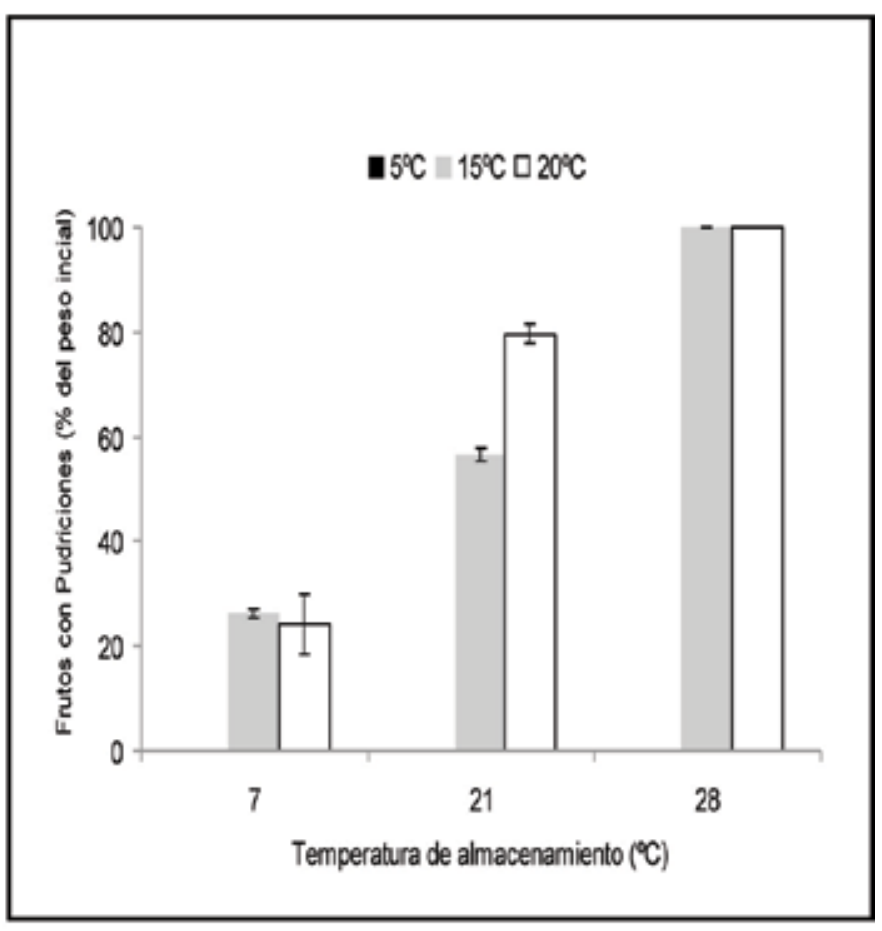

D.

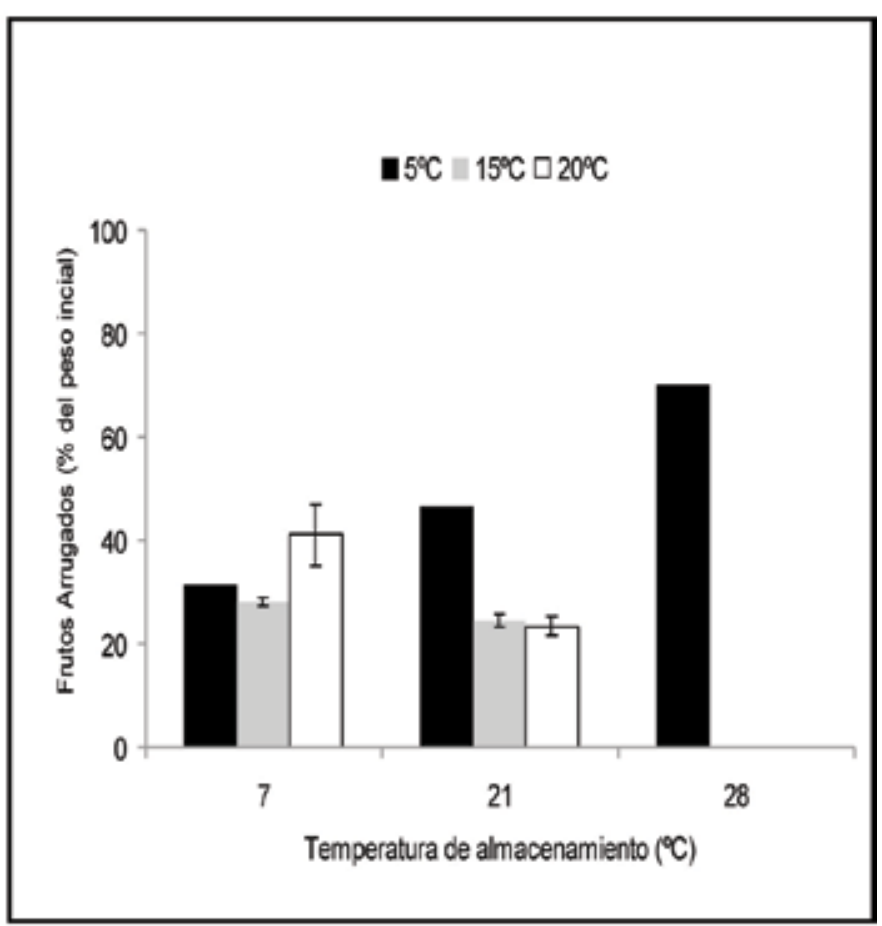

Figura 4. Frutos Sin Defectos (A), con Pudriciones (B), Arrugados (C) y Manchados (D), en tres temperaturas de conservación (5, 15 y $20^{\circ} \mathrm{C}$ ) y en cada momento de salida del almacenamiento (7,21 y 28 días), expresado en porcentaje del peso inicialmente almacenado. 


\section{Color}

En este estudio no se observaron cambios de brillo (luminosidad) relevantes en la cáscara ni en la pulpa. Al mismo tiempo, el tono de color de la pulpa tendió a ser menos anaranjado y más amarillo a los 28 días de conservación a $5{ }^{\circ} \mathrm{C}$, y menos saturado a mayor temperatura y tiempo de conservación (Tabla 1). El color de la cáscara de los frutos fue anaranjado más intenso a los 21 días de conservado a $5^{\circ} \mathrm{C}$, posiblemente debido a un incremento de las coloraciones más oscuras (marrones) que dieron lugar a manchas. A su vez, la pulpa tuvo un índice de color más claro (más amarillo, menos anaranjado) que la cáscara. Los cambios de color pueden estar asociados a la pérdida de pigmentos (carotenoides y flavonoides) y/o a procesos de oxidación enzimática (Saltveit, 2000). Sin embargo, como se describirá más adelante, el menor color anaranjado no se explicaría en este estudio por pérdida de trans- $\beta$-caroteno.

\begin{tabular}{|c|c|c|c|c|c|c|c|c|}
\hline \multicolumn{5}{|c|}{ Cáscara } & \multicolumn{4}{|c|}{$\overline{P u l p a}$} \\
\hline & $\mathbf{0}$ & 7 & 21 & 28 & $\mathbf{0}$ & 7 & 21 & 28 \\
\hline \multicolumn{9}{|c|}{ Luminosidad (L) } \\
\hline $5^{\circ} \mathrm{C}$ & $42,6 \pm 0,3$ & $43,7 \pm 0,3 \mathrm{a}$ & $41,7 \pm 0,3$ & $42,6 \pm 0,2$ & $42,6 \pm 0,3$ & $43,7 \pm 0,3 \mathrm{a}$ & $41,7 \pm 0,3$ & $42,6 \pm 0,2$ \\
\hline $15^{\circ} \mathrm{C}$ & $42,6 \pm 0,3$ & $42,7 \pm 0,7 b$ & - & - & $42,6 \pm 0,3$ & $42,7 \pm 0,7 b$ & - & - \\
\hline $20^{\circ} \mathrm{C}$ & $42,6 \pm 0,3$ & $42,9 \pm 0,4 a$ & - & - & $42,6 \pm 0,3$ & $42,9 \pm 0,4 a$ & - & - \\
\hline \multicolumn{3}{|c|}{ * Temperatura $\geq 0,0133$} & \multicolumn{2}{|c|}{ Momento $\geq 0,1351$} & \multicolumn{2}{|c|}{ Temperatura $\mathrm{P} \leq 0,2337$} & \multicolumn{2}{|c|}{ Momento $\mathrm{P} \leq 0,0190$} \\
\hline \multicolumn{9}{|c|}{ Tono ('hue) } \\
\hline $5^{\circ} \mathrm{C}$ & $68,4 \pm 1,1$ & $69,2 \pm 0,6 \mathrm{a}$ & $67,5 \pm 0,7$ & $68,3 \pm 5,3$ & $76,7 \pm 1,7 \mathrm{~B}$ & $76,2 \pm 0,6 \mathrm{aA}$ & $78,1 \pm 0,8 \mathrm{AB}$ & $80,5 \pm 0,5 \mathrm{C}$ \\
\hline $15^{\circ} \mathrm{C}$ & $68,4 \pm 1,1$ & $71,1 \pm 0,4 b$ & - & - & $76,7 \pm 1,7$ & $75,6 \pm 1,8 \mathrm{a}$ & - & - \\
\hline $20^{\circ} \mathrm{C}$ & $68,4 \pm 1,1$ & $69,2 \pm 0,4 \mathrm{a}$ & - & - & $76,7 \pm 1,7$ & $80,4 \pm 0,6 \mathrm{~b}$ & - & - \\
\hline \multicolumn{3}{|c|}{ Temperatura $\geq 0,0049$} & \multicolumn{2}{|c|}{ Momento $\geq 0,1406$} & \multicolumn{2}{|c|}{ Temperatura $\mathrm{P} \leq 0,0074$} & \multicolumn{2}{|c|}{ Momento $\mathrm{P} \leq 0,0001$} \\
\hline \multicolumn{9}{|c|}{ Saturación (Croma) } \\
\hline $5^{\circ} \mathrm{C}$ & $48,8 \pm 5,2 \mathrm{~A}$ & $45,1 \pm 0,4 \mathrm{aB}$ & $45,7 \pm 0,7 \mathrm{~B}$ & $45,4 \pm 0,3 \mathrm{~B}$ & $48,6 \pm 1,5 \mathrm{~A}$ & $51,2 \pm 0,6 \mathrm{aB}$ & $48,6 \pm 0,5 \mathrm{a}$ & $52,7 \pm 0,4 \mathrm{~B}$ \\
\hline $15^{\circ} \mathrm{C}$ & $48,8 \pm 5,2$ & $46,8 \pm 0,5 b$ & - & - & $48,6 \pm 1,5$ & $51,9 \pm 1,4 \mathrm{a}$ & - & - \\
\hline $20^{\circ} \mathrm{C}$ & $48,8 \pm 5,2$ & $45,9 \pm 0,4 \mathrm{ab}$ & - & - & $48,6 \pm 1,5$ & $62,9 \pm 0,6 \mathrm{~b}$ & - & - \\
\hline \multicolumn{3}{|c|}{ Temperatura $\leq 0,0214$} & \multicolumn{2}{|c|}{ Momento $\leq 0,0001$} & \multicolumn{2}{|c|}{ Temperatura $\mathrm{P} \leq 0,0001$} & \multicolumn{2}{|c|}{ Momento $\mathrm{P} \leq 0,0001$} \\
\hline \multicolumn{9}{|c|}{ Índice de Color } \\
\hline $5^{\circ} \mathrm{C}$ & $9,1 \pm 2,5$ & $9,1 \pm 2,4$ & $10,1 \pm 2,6$ & $9,4 \pm 2,6$ & $4,6 \pm 0,9 \mathrm{aA}$ & $5,3 \pm 1,5 \mathrm{aA}$ & $4,8 \pm 1,7 \mathrm{a}$ & $3,6 \pm 1,2 \mathrm{~B}$ \\
\hline $15^{\circ} \mathrm{C}$ & $9,1 \pm 2,5$ & $8,0 \pm 1,9$ & - & - & $4,6 \pm 0,9 \mathrm{a}$ & $4,8 \pm 1,0 \mathrm{a}$ & - & - \\
\hline $20^{\circ} \mathrm{C}$ & $9,1 \pm 2,5$ & $8,9 \pm 1,9$ & - & - & $4,6 \pm 0,9 \mathrm{a}$ & $3,5 \pm 1,2 b$ & - & - \\
\hline \multicolumn{3}{|c|}{ Temperatura $\geq 0,1149$} & \multicolumn{2}{|c|}{ Momento $\leq 0,0988$} & \multicolumn{2}{|c|}{ Temperatura $\mathrm{P} \leq 0,0001$} & \multicolumn{2}{|c|}{ Momento $\mathrm{P} \leq 0,0001$} \\
\hline
\end{tabular}

Tabla 1. Luminosidad, Tono ('hue), Saturación (Croma) e Índice de color en cáscara y pulpa de butiá conservados a 5,15 y $20^{\circ} \mathrm{C}$ durante $0,7,21$ y 28 días. Media $\pm E E$. Letras minúsculas iguales dentro de la misma columna o mayúsculas dentro de la fila indican que no hay diferencia estadística * Valor P de efectos principales (Temperatura y Momento).

\section{Materia seca, sólidos solubles totales, pH y acidez titulable}

El contenido de materia seca (MS) de la cáscara fue similar en todas las temperaturas y tiempos de conservación con valores entre 18,9 y $22,3 \%$ (Tabla 3 ). El contenido de MS de la pulpa fue menor que el de la cáscara, y se modificó con la temperatura de conservación. A $5{ }^{\circ} \mathrm{C}$ el contenido de MS estuvo entre 15,5 y $16,4 \%$, y temperaturas de 15 y $20^{\circ} \mathrm{C}$ de conservación determinaron menor contenido de MS. Los sólidos solubles (SST) en el jugo de pulpa de butiá disminuyeron con el tiempo de conservación y al aumentar la temperatura. A la semana de almacenamiento se observó que elevar la temperatura de conservación $10^{\circ} \mathrm{C}\left(\right.$ de $5{ }^{\circ} \mathrm{C}$ a $\left.15^{\circ} \mathrm{C}\right)$ provocó una pérdida de 30 a $40 \%$ de SST. El contenido de MS y de SST obtenidos en pulpa son similares a los reportados en pulpa de butiá por Faria et al. (2008) y Crosa et al. (2011). La disminución del contenido de MS y de SST al aumentar el tiempo y la temperatura de almacenamiento puede explicarse por la mayor respiración, el aumento de la permeabilidad de la membrana celular y la degradación de las paredes celulares (Salunkhe y Dasai, 1984; Saltveit, 2000).

$\mathrm{Al}$ igual que ha sido reportado por Faria et al. (2008) y Crosa et al. (2011) se obtuvo un pH del jugo de frutos de butiá muy ácido. Al mismo tiempo, se observó que el porcentaje de ácido cítrico, determinado por acidez titulable, bajó al aumentar el tiempo y la temperatura de conservación (Tabla 2).

\begin{tabular}{|c|c|c|c|c|}
\hline & $\mathbf{0}$ & 7 & 21 & 28 \\
\hline \multicolumn{5}{|c|}{ SST ( ${ }^{\circ}$ Brix) } \\
\hline $5^{\circ} \mathrm{C}$ & $14,8 \pm 0,7 \mathrm{~A}$ & $13,2 \pm 0,6 \mathrm{aB}$ & $11,8 \pm 0,3 \mathrm{~B}$ & $10,1 \pm 0,2 \mathrm{C}$ \\
\hline $15^{\circ} \mathrm{C}$ & $14,8 \pm 0,7$ & $8,9 \pm 0,5 b$ & - & - \\
\hline $20^{\circ} \mathrm{C}$ & $14,8 \pm 0,7$ & $8,7 \pm 0,4 b$ & - & - \\
\hline \multicolumn{3}{|c|}{ Temperatura $\mathrm{P} \leq 0,0001$} & \multicolumn{2}{|c|}{ Momento $\leq 0,0001$} \\
\hline \multicolumn{5}{|c|}{$\mathrm{pH}$} \\
\hline $5^{\circ} \mathrm{C}$ & $2,54 \pm 0,01$ & $3,15 \pm 0,09 \mathrm{aB}$ & $4,97 \pm 0,15 \mathrm{~A}$ & $3,22 \pm 0,01 \mathrm{~B}$ \\
\hline $15^{\circ} \mathrm{C}$ & $2,56 \pm 0,01$ & $2,86 \pm 0,04 b$ & - & - \\
\hline $20^{\circ} \mathrm{C}$ & $2,54 \pm 0,01$ & $3,31 \pm 0,01 \mathrm{a}$ & - & - \\
\hline \multicolumn{3}{|c|}{ Temperatura $\mathrm{P} \leq 0,0031$} & \multicolumn{2}{|c|}{ Momento $\leq 0,0001$} \\
\hline \multicolumn{5}{|c|}{ Acidez titulable (\% ácido cítrico) } \\
\hline $5^{\circ} \mathrm{C}$ & $1,60 \pm 0,01 \mathrm{~B}$ & $2,02 \pm 0,02 \mathrm{aA}$ & $1,37 \pm 0,01 \mathrm{C}$ & $1,37 \pm 0,01 \mathrm{C}$ \\
\hline $15^{\circ} \mathrm{C}$ & $1,60 \pm 0,01$ & $1,48 \pm 0,01 b$ & - & - \\
\hline $20^{\circ} \mathrm{C}$ & $1,60 \pm 0,01$ & $1,28 \pm 0,11 \mathrm{c}$ & - & - \\
\hline \multicolumn{3}{|c|}{ Temperatura $\mathrm{P} \leq 0,0031$} & \multicolumn{2}{|c|}{ Momento $\leq 0,0001$} \\
\hline
\end{tabular}

Tabla 2. Sólidos solubles totales ( ${ }^{\circ} \mathrm{Brix}$ ), $\mathrm{pH}$; acidez titulable (\% ácido cítrico) en jugo de pulpa de butiá conservados a 5, 15 y $20^{\circ} \mathrm{C}$ durante $0,7,21$ y 28 días. Media \pm EE. Letras minúsculas iguales dentro de la misma o mayúsculas dentro de la fila indican que no hay diferencia estadística *Valor $\mathrm{P}$ de efectos principales (Temperatura y Momento). 


\begin{tabular}{|c|c|c|c|c|c|c|c|c|}
\hline \multicolumn{4}{|c|}{ Cáscara } & \multirow[b]{2}{*}{28} & \multicolumn{4}{|c|}{ Pulpa } \\
\hline & $\mathbf{0}$ & 7 & 21 & & $\mathbf{0}$ & 7 & 21 & 28 \\
\hline \multicolumn{9}{|c|}{ Materia Seca (\%) } \\
\hline $15^{\circ} \mathrm{C}$ & $21,3 \pm 0,1$ & $18,6 \pm 0,7$ & - & - & $16,2 \pm 0,1$ & $12,3 \pm 0,4 \mathrm{~b}$ & - & - \\
\hline $20^{\circ} \mathrm{C}$ & $21,3 \pm 0,1$ & $18,9 \pm 0,8$ & - & - & $16,2 \pm 0,1$ & $13,2 \pm 0,6 b$ & - & - \\
\hline \multicolumn{3}{|c|}{ Temperatura $P \geq 0,2186$} & \multicolumn{2}{|c|}{ Momento $\mathrm{P} \geq 0,266$} & \multicolumn{2}{|c|}{ Temperatura $\mathrm{P} \leq 0,0001$} & \multicolumn{2}{|c|}{ Momento $\mathrm{P} \geq 0,0697$} \\
\hline \multicolumn{9}{|c|}{ trans-ß-caroteno $(\mathrm{mg} / \mathbf{1 0 0 g}$ peso fresco) } \\
\hline $5^{\circ} \mathrm{C}$ & $1,44 \pm 0,01$ & $1,85 \pm 0,03$ & $2,10 \pm 0,31$ & $2,05 \pm 0,26$ & $1,06 \pm 0,01 \mathrm{a}$ & $0,76 \pm 0,03 b$ & $1,15 \pm 0,25 \mathrm{a}$ & $1,03 \pm 0,06 \mathrm{a}$ \\
\hline $15^{\circ} \mathrm{C}$ & $1,44 \pm 0,01$ & $2,32 \pm 0,15$ & - & - & $1,06 \pm 0,01$ & $0,96 \pm 0,15$ & - & - \\
\hline $20^{\circ} \mathrm{C}$ & $1,44 \pm 0,01$ & $2,36 \pm 0,12$ & - & - & $1,06 \pm 0,01$ & $0,81 \pm 0,12$ & - & - \\
\hline \multicolumn{3}{|c|}{ Temperatura $\mathrm{P} \leq 0,138$} & \multicolumn{2}{|c|}{ Momento $\mathrm{P} \leq 0,257$} & \multicolumn{2}{|c|}{ Temperatura $\mathrm{P} \leq 0,780$} & \multicolumn{2}{|c|}{ Momento $\mathrm{P} \leq 0,029$} \\
\hline
\end{tabular}

Tabla 3. Materia seca (\%) y contenido de trans- $\beta$-caroteno (mg/100 g peso fresco) en cáscara y pulpa de butiá conservados a 5 , 15 y $20{ }^{\circ} \mathrm{C}$ durante $0,7,21$ y 28 días. Media \pm EE. Para la cáscara o para la pulpa, letra dentro de la misma columna indican que no hay diferencias estadísticas (Tukey $\geq 0,05$ ). ${ }^{\star}$ Valor $\mathrm{P}$ de efectos principales (Temperatura y Momento).

\section{Contenido de trans- $\beta$-caroteno}

En las condiciones de este trabajo no se observaron modificaciones en el contenido de trans- $\beta$-caroteno en la cáscara por efecto de la temperatura y tiempo de conservación. El contenido trans- $\beta$-caroteno de la pulpa fue sensiblemente menor a los siete días de conservación a $5{ }^{\circ} \mathrm{C}$ (Tabla 3). En todos los casos se cuantificó mayor cantidad de trans- $\beta$-caroteno en cáscara que en pulpa. La estructura celular y la composición de la cáscara es diferente a la pulpa, probablemente con mayor contenido de ceras y cloroplastos a los cuales están asociados los carotenoides (Paliyath y Murr, 2008). Los frutos de butiá han sido reportados como una buena fuente de carotenoides, con 1,11 a $4,39 \mathrm{mg} / 100 \mathrm{~g}$ de pulpa fresca, con el $\beta$-caroteno dominante con 1,61 $\mathrm{mg} / 100 \mathrm{~g}$, seguido de otros carotenoides no pro-vitamínicos como fitoeno y fitoflueno (Faria et al., 2011). A partir de los resultados obtenidos se puede estimar que la cantidad de $\beta$-caroteno aportados por $100 \mathrm{~g}$ de butiá enteros in natura (cáscara y pulpa) cubriría el 16,4 \% de los requerimientos diarios de un niño (WHO/FAO, 2003).

En las condiciones de este estudio se concluye que el butiá puede conservarse a $5{ }^{\circ} \mathrm{C}$ durante siete días, manteniendo características de color, MS, $\mathrm{pH}$ y contenido de trans- $\beta$-caroteno para consumo en fresco (in natura) o para su industrialización. La cantidad de frutos que se mantiene con calidad a la semana de conservación a $5{ }^{\circ} \mathrm{C}$ es bajo ( $47 \%$ del peso inicialmente almacenado), lo que verifica que es un fruto con alta tasa de deterioro. Para disminuir el deterioro de los frutos para su uso entero in natura, en el momento de la cosecha debe reducirse rápidamente la temperatura de pulpa (a menos de $5{ }^{\circ} \mathrm{C}$ ); la temperatura de conservación óptima es menor a $5{ }^{\circ} \mathrm{C}$. Asimismo, se debe considerar realizar modificaciones fundamentales en el proceso de cosecha de los frutos, que minimicen su daño físico. La información obtenida ha permitido caracterizar la evolución de atributos físicos y químicos de butiá en las condiciones de cosecha estudiadas y durante su almacenamiento en tres temperaturas.

\section{Reconocimientos}

A la Gerencia de Proyectos Alimentarios y al equipo técnico del Departamento de Gestión y Transferencia Tecnológica para el Desarrollo Local del LATU, Carlos Ayres y Julio Sosa. Al Sr. Molina (cosechador) por sus aportes de conocimientos durante la cosecha de los frutos. A Roxana Las por realizar parte de los trabajos de análisis en el Laboratorio de Calidad de Alimentos y de Calidad de Productos de la Facultad de Agronomía. Este trabajo fue financiado por el INIA a través del Proyecto FPTA 178.

\section{Referencias}

- AMARANTE, Cassandro Talamini do; MEGGUER, Clarice Aparecida. Qualidade pós-colheita de frutos de butiá em funçao do estádo de maturaçao na colheita e do manejo da temperatura. En: Ciencia Rural. 2008, 38(1):46-53.

- BAEZ, Fernando; JAURENA, Martín. Regeneración del palmar de butiá (Butia capitata) en condiciones de pastoreo: relevamiento de establecimientos rurales de Rocha. Rocha: PROBIDES, 2000.

(Documentos de Trabajo; 27)

- BARTZ, Jerry A.; BRECHT, Jeffrey K. Postharvest physiology and pathology of vegetables. 2a. ed. New York: Marcel Dekker, 2003. (Food Science and Technology; 123).

- BETANCURT, Pablo; GIOSCIA, Diego; AYRES, Carlos; ARCIA, Patricia. Frutos autóctonos de butiá: innovación y transferencia tecnológica. En: INNOTEC. 2008, (3):63-71.

- BURZACO, Patricia; IRISITY, Mariana; RUIZ-DÍAZ, María Fernanda; SOSA, Julio; PINTO, Ángel. Bombones. Montevideo: LATU, 2011. (Serie Frutos Nativos: Butiá; 5).

- CROSA, María José; BURZACO, Patricia; PASTORINO, N.; IRISITY, Mariana; GIOSCIA, Diego; AYRES, Carlos.

Caracterización fisicoquímica y nutricional del fruto Butia capitata y de su pulpa tamizada. En: INNOTEC. 2011, (6):3-6.

- FARIA, Juliana Pereira; ARELLANO, Daniel Barrera; GRINALDI, Renato; SILVA, Lucas Carvalho Ramos da; VIEIRA, Roberto Fontes; SILVA, Dijalma Barboza; AGOSTINI-COSTA, Tania da Silveira. Caracterização da polpa do coquinho-azedo (Butia capitata var capitata). En: Revista Brasileira Fruticultura. 2008, 30(3):827-829.

- FARIA, Juliana Pereira; SIQUEIRA, Egle; VIEIRA, Roberto Fontes; AGOSTINI-COSTA, Tania Da Silveira. Fruits of Butia capitata (Mart.) as good sources of $\beta$-carotene and provitamina. En: Revista Brasileira Fruticultura. 2011, Volumen Especial:612-617.

- JIMÉNEZ, M.; CUQUERELLA, J.; MARTINEZ-JAVEGA, José María. Determination of color index for citrus fruits degreening. En: Proceeding International Society Citriculture. 1981, (2):750753.

- NEGI, Preadeep; HANDA, Avtar. Structural deterioration of the produce: the breakdown of cell components. En: PALIYATH, Gophinadhan; MURR, Dennis; HANDAR, Avtar; LURIE, Susan. 
Postharvest biology and technology fruits, vegetables and flowers. San Francisco: Wiley-Blackwell, 2008. pp.162-194.

- PALIYATH, Gophinadhan; MURR, Dennis. Biochemistry of fruits. En: PALIYATH, Gophinadhan; MURR, Dennis; HANDAR, Avtar; LURIE, Susan. Postharvest biology and technology fruits, vegetables and flowers. San Francisco: Wiley-Blackwell, 2008. pp.19-50.

- RIVAS, Mercedes. Conservación in situ de recursos fitogenéticos. En: IICA. Estrategias en recursos fitogenéticas para países del Cono Sur. Montevideo: IICA; PROCISUR, 2001. pp. 65-78.

- RIVAS, Mercedes; BARILANI, A. Diversidad, potencial productivo y reproductivo de los palmares de Butia capitata (Marc.) Becc. de Uruguay. En: Agrociencia. 2004, (8):11-20.

- SALTVEIT, Mikael. Wound induced changes in phenolic metabolism and tissue browning are altered by heat shock. En: Postharvest Biology and Technology. 2000, (21):61-69.

- SALUNKHE, D.; DESAI, B. Post harvest biotechnology of vegetables. Vol. 1. Boca Raton: CRC Press, 1984.

- SCHWARTZ, E.; NEITTZEL, R.S.; BARBIERI, R.L.; FACHUNELLO, J.C. Variabilidade em inflorescências de butiazeiros de Santa Vitória do Palmar. En: EMBRAPA. III Simpósio Nacional do Morango. II Encontro sobre Pequenas Frutas e Frutas Nativas do Mercosul, (Pelotas 7 a 10 de noviembre de 2006). Pelotas : Embrapa, 2007. (Documentos 203) pp. 250252.

- SZPYLKA, John; DE VRIES, Jonathan W. Determination of carotene in supplements and raw materials by reversed-phase high pressure liquid chromatography: collaborativee study. En : Journal of AOAC International. 2005, (88):1279-1291.

- WHO; FAO. Diet, nutrition and the prevention of chronic diseases. Report of a joint WHO/FAO expert consultation. Geneve: WHO; FAO, 2003. (WHO Technical Report Series; 916)

- ZACCARI, Fernanda. Caracterización de seis cultivares de zanahorias (Daucus carota, L.), crudas y cocidas al vapor, por color y contenido y bioaccesibilidad in vitro de $\beta$-carotenos $y$ minerales. Montevideo: UDELAR. Facultad de Agronomía, 2010. (Tesis de Maestría). 